\title{
Molecular Basis of ABO Variants Including Identification of 16 Novel ABO Subgroup Alleles in Chinese Han Population
}

\author{
Yan-ling Ying ${ }^{a-c}$ Xiao-zhen Hong ${ }^{b, c}$ Xian-guo Xu ${ }^{b, c}$ Shu Chen ${ }^{b, c} J_{i} \mathrm{He}^{\mathrm{b}, \mathrm{c}}$ \\ Fa-ming Zhu ${ }^{\text {b, c }}$ Xin-you Xie ${ }^{a}$ \\ ${ }^{a}$ Sir Run Run Shaw Hospital, Zhejiang University School of Medicine, Hangzhou, PR China; ${ }^{b}$ Blood Center of \\ Zhejiang Province, Hangzhou, PR China; ' Key Laboratory of Blood Safety Research of Zhejiang Province, Hangzhou, \\ PR China
}

\section{Keywords}

$\mathrm{ABO}$ variants $\cdot$ Molecular basis $\cdot$ Novel $A B O$ allele . Sequence-based typing

\begin{abstract}
Introduction: The characteristic of $\mathrm{ABO}$ blood subgroup is crucial for elucidating the mechanisms of such variant phenotypes and offering useful information in blood transfusion. Methods: In total, $211 \mathrm{ABO}$ variants including part of available family members were investigated in this study. The phenotypes of these individuals were typed with serologic methods. The full coding regions of $A B O$ gene and the erythroid cellspecific regulatory elements in intron 1 of them were amplified with polymerase chain reaction and then directly sequenced. The novel alleles were confirmed by cloning and sequencing. Phylogenetic tree was made using CLUSTAL W software. 3D structural analyses of the glycosyltransferases (GTs) with some typical mutations were performed by PyMOL software. Results: Forty-eight distinctly rare $A B O$ alleles were identified in 211 Chinese variant individuals, including 16 novel $A B O$ alleles. All of the alleles were categorized as 5 groups: $16 A B O^{*} A$ alleles, $23 A B O^{*} B$ alleles, $4 A B O^{*} B A$ alleles, $4 A B O^{*} c i s A B$ alleles, and $1 A B O^{*} O$ alleles. $A B O^{*} A 2.08$ and $A B O^{*} B A .02$ were the relatively predominant $A$ and $B$ subgroup alleles, respectively. According to the phylogenetic tree, 28 alleles (5 common alleles and 23 alleles identified in our laboratory) were classified into 3 major allelic lineages. The struc-
\end{abstract}

Xinyou Xie and Faming Zhu contributed equally to the manuscript. tural analysis of 3D homology modeling predicted reduced protein stability of the mutant GTs and may explain the reduced $A B O$ antigen expression. Conclusions: The molecular basis of $A B O$ variants was analyzed, and 16 novel $A B O$ alleles were identified. The results extended the information of $A B O$ variants and provided a basis for better transfusion strategies and helped to improve blood transfusion safety.

C 2019 S. Karger AG, Basel

\section{Introduction}

ABO is considered one of the most complicatedly and clinically relevant blood group system in the fields of both transfusion and transplantation medicine [1]. More than 300 different $A B O$ alleles have been characterized to date. Except for the 5 common $A B O$ alleles $\left(A B O^{*} A 1.01\right.$, $A B O^{*} A 1.02, A B O^{*} B .01, A B O^{*} 0.01 .01$, and $\left.A B O^{*} 0.01 .02\right)$ [2], numerous subgroup allele-related variants with a differential expression of the A or B antigens on the red blood cells have also been found in different populations [3-5]. ABO variant prevalence is rare, with a rate of approximately $0.015 \%$ in China [6]. The molecular genetic basis of the $\mathrm{ABO}$ system had been known since the $A B O$ cDNA corresponding to the mRNA was cloned in 1990 [7]. Many ABO variants caused by the mutation in the coding region had been identified, although the molecular mechanisms underlying some variants were still not 
understood completely. Several genetic events, including substitution, splice site mutation, base insertion, deletion, and hybrid alleles, have the potential to affect the A or B glycosyltransferase (GT) activity and then cause changes in $\mathrm{ABO}$ phenotypic expression $[8,9]$. Besides the changes of the exons, the erythroid cell-specific regulatory elements of the $A B O$ gene located in the $5^{\prime}$ - and $3^{\prime}$-flanking sequence and the first intron 1 were found to affect the transcription efficiency [10-13]. The distribution and diversity of $A B O$ variants were crucial for elucidating phenotype exactly and offering useful information for blood transfusion. In this study, some apparent $\mathrm{ABO}$ variants including part of available family members were investigated for the serology characterization by serological agglutination reaction method, and then the molecular basis was further analyzed by polymerase chain reaction sequence-based typing (PCR-SBT) method and 3D structural prediction. The following report described the characteristics of $A B O$ variants in Chinese population.

\section{Materials and Methods}

\section{Study Samples}

A total of $211 \mathrm{ABO}$ variant samples were collected, including the volunteer blood donors in Blood Center of Zhejiang Province, China, the patients in the hospitals of Hangzhou City in Zhejiang Province, and part of the related family members. All of the individuals were collected from unselected population and are not the same. All individuals were found with discrepancies in ABO red cell grouping and serum grouping, or weak A and/or B antigen expression.

Venous blood of these $\mathrm{ABO}$ variant samples was collected. Genomic DNAs were extracted from blood cells using a commercial DNA whole blood kit with a nucleic acid isolation device (QuickGeneMini80, FujiFilm Corporation, Tokyo, Japan) according to the manufacturer's instruction.

\section{Serologic Typing}

$\mathrm{ABO}$ phenotypes were determined by agglutination and adsorption-elution tests and serologic diagnostic classification according to standard methods and procedures described in $\mathrm{AABB}$ technical manual [14]. The following commercially available reagents were used: monoclonal anti- $\mathrm{A}$, anti- $\mathrm{B}$, anti-AB (Shanghai Hemo-pharmaceutical Biological Company, Shanghai, China), Anti-A1 (Dolichos Biflorus lectin), and Anti-H (Ulex europeus; Dominion Company, Dartmouth, Canada).

\section{PCR Amplification and Sequencing for Full Coding Region of} ABO Gene

The full coding regions of exons 1-7 of $A B O$ gene were amplified with 3 pairs of primers according to our previous reports [ 15 , 16]. PCR amplification products were purified with double enzymes digestion and sequenced bidirectionally using a Bigdye Terminator Cycle version 3.1 sequencing kit (Applied Biosystems, Foster City, CA, USA) [17]. The sequence data were analyzed by Seqscape2.5 software (Applied Biosystems, Foster City, CA, USA) and assigned for the $A B O$ genotype according to the reference sequence of $A B O^{*} A 1.01$ gene (AC000397) polymorphism positions [4].

Molecular Basis of ABO Blood Group

\section{Haplotype Analysis of Novel ABO Alleles}

To confirm the novel $A B O$ alleles, PCR amplified fragments containing the novel mutation were directly ligated and cloned into the plasmid pCR4-TOPO (Invitrogen, Carlsbad, CA, USA) according to the manufacturer's instruction. Plasmid DNA was extracted from each recombinant colony with 3S Spin Plasmid Miniprep Kits (Shenergy Biocolor, Shanghai, China) and used as the template for sequencing reactions according to our previous reports $[15,17]$.

\section{PCR Amplification and Sequencing for the 5.8-kb Region in}

\section{Intron 1}

The individuals without any subgroup alleles were analyzed for the nucleotide sequences of the partial intron 1 covering the +5.8$\mathrm{kb}$ region. The method of the amplification and direct sequencing is referred to in our previous reports [10]. The nucleotide sequences were compared with standard $A B O$ polymorphism site sequences (NT_035014).

\section{Nomenclature of Mutation and ABO Alleles}

The new allele sequences have been submitted to the GenBank Database, and part of $A B O$ alleles have been named according to the nomenclature by ISBT (http://www.isbtweb.org/working-parties/red-cellimmunogenetics-and-blood-group-terminology/) [3]

\section{Dendrogram Construction}

The sequences were aligned and phylogenetic tree was constructed using CLUSTAL W software. Phylogenetic trees showing the phylogenetic relationship between the alleles were constructed according to the neighbor-joining method [18].

\section{Modeling of Some Typical Novel Alleles}

To understand the effect of mutations on the structure or activity of GTs produced from the novel alleles, 3D molecular models of some typical novel mutations were generated from the template structure of wild-type A glycosyltransferases (GTAs) (PDB code, 1LZI) and B glycosyltransferases (GTBs) (PDB code, 1LZ7) by the PyMOL software.

\section{Results}

\section{The Serological Characteristic of ABO Variant Phenotypes}

A total of $211 \mathrm{ABO}$ variant phenotypes could be fit into the following categories: $A_{2}, A_{2} B, A_{x}, A_{x} B, A_{m} B$, $A_{e l}, A_{e l} B, B_{3}, B_{x}, A B_{x}, B_{m}, B_{e l}, A B_{w}$, and $O$. Reducing of $A$ or $B$ antigen was found in most cases. Meanwhile, part of individuals with normal antigen expression had lack or superfluous amount of antibody in the serum. Some individuals were just classified as $A_{w} / A_{w} B$ or $B_{w} / A B_{w}$ because of the discrepancy between the serological characteristic and standard. The detail serologic characterizations of these variants were listed in Table 1 .

\section{Molecular Characterization of $A B O$ Variants}

In all, 48 rare $A B O$ alleles responsible for the $\mathrm{ABO}$ subgroup phenotypes were found in 136 variants by the di- 
Table 1. The serologic characterization of samples with rare ABO subgroup alleles found in this study

\begin{tabular}{|c|c|c|c|c|c|c|c|c|c|c|c|}
\hline \multirow[t]{2}{*}{ Phenotype } & \multirow[t]{2}{*}{$\mathrm{NO}^{\mathrm{a}}$} & \multicolumn{5}{|c|}{ Cell group } & \multicolumn{3}{|c|}{ Reverse (serum) grouping } & \multicolumn{2}{|c|}{$\begin{array}{l}\text { Absorption and } \\
\text { elution test }\end{array}$} \\
\hline & & anti-A & anti-B & anti-AB & anti-A1 & anti-H & $\overline{A c}$ & $\mathrm{Bc}$ & Oc & $\overline{A c}$ & $\mathrm{Bc}$ \\
\hline A2 & 9 & $4+^{\mathrm{b}}$ & 0 & $4+$ & 0 & $4+$ & $0 \sim 3+$ & $4+$ & 0 & $j^{\mathrm{c}}$ & l \\
\hline $\mathrm{A} 2 \mathrm{~B}$ & 20 & $4+$ & $4+$ & $4+$ & 0 & $1+\sim 4+$ & $0 \sim 3+$ & 0 & 0 & l & l \\
\hline $\mathrm{Ax}$ & 4 & + & 0 & $1+\sim 3+$ & 0 & $2+\sim 4+$ & $1+$ & $4+$ & 0 & l & l \\
\hline $\mathrm{AxB}$ & 7 & $1+$ & $4+$ & $4+$ & 0 & $2+$ & $1+\sim 2+$ & 0 & 0 & l & l \\
\hline Ael & 9 & 0 & 0 & 0 & 0 & $4+$ & $1+\sim 2+$ & $4+$ & 0 & $3+\sim 4+$ & 0 \\
\hline $\mathrm{A}_{\mathrm{w}}$ & 3 & $\pm \sim 2+$ & 0 & $1+\sim 2+$ & 0 & $3+\sim 4+$ & $0 \sim 1+$ & $4+$ & 0 & I & I \\
\hline $\mathrm{A}_{\mathrm{w}} \mathrm{B}$ & 21 & $2+\sim 3+$ & $4+$ & $4+$ & 0 & $2+\sim 4+$ & $0 \sim 1+$ & 0 & 0 & 1 & 1 \\
\hline B3 & 6 & 0 & $\mathrm{mf}$ & $\mathrm{mf}$ & / & $4+$ & $4+$ & 0 & 0 & 1 & 1 \\
\hline$B x$ & 7 & 0 & $1+\sim 2+$ & $1+\sim 2+$ & I & $4+$ & $4+$ & 0 & 0 & 1 & I \\
\hline $\mathrm{ABx}$ & 5 & $4+$ & $1+\sim 2+$ & $4+$ & I & $1+\sim 2+$ & 0 & 0 & 0 & 1 & I \\
\hline Bel & 3 & 0 & 0 & 0 & I & $4+$ & $4+$ & $2+\sim 3+$ & 0 & 0 & $3+\sim 4+$ \\
\hline $\mathrm{B}_{\mathrm{m}}$ & 2 & 0 & $1+$ & $1+$ & I & $4+$ & $4+$ & 0 & 0 & 1 & I \\
\hline $\mathrm{B}_{\mathrm{w}}$ & 20 & 0 & $1+\sim 3+$ & $2+\sim 3+$ & I & $4+$ & $4+$ & $\pm \sim 1+$ & 0 & I & I \\
\hline $\mathrm{AB}_{\mathrm{w}}$ & 19 & $4+$ & $1+\sim 3+$ & $4+$ & $4+$ & $2+$ & 0 & $\pm \sim 3+$ & 0 & I & I \\
\hline
\end{tabular}

a The number of the sample with rare ABO subgroup alleles identified in this study. ${ }^{\mathrm{b}}$ The " + " denotes the agglutination strength of serology. ${ }^{c}$ The "/" denotes that the reaction has not been done.

Table 2. Sixteen novel $A B O$ subgroup alleles identified in our laboratory in this study

\begin{tabular}{|c|c|c|c|c|c|}
\hline Allele ${ }^{\mathrm{a}}$ & Critical nucleotide ${ }^{\mathrm{b}}$ & $\begin{array}{l}\text { Main amino acid } \\
\text { change(s) }\end{array}$ & $\begin{array}{l}\text { Number } \\
(\mathrm{R})^{\mathrm{c}}\end{array}$ & Phenotype & $\begin{array}{l}\text { GenBank } \\
\text { number }\end{array}$ \\
\hline$A B O^{*} A 2.10$ & $268 \mathrm{~T}>\mathrm{C} ; 467 \mathrm{C}>\mathrm{T}$ & W90R; P156L & 1 & $\mathrm{~A}_{2}$ & $-^{\mathrm{e}}$ \\
\hline$A B O^{*} A 2.11$ & $266 \mathrm{C}>\mathrm{T} ; 467 \mathrm{C}>\mathrm{T}$ & P89L; P156L & 3 & $\mathrm{~A}_{2} \mathrm{~B}$ & - \\
\hline$A B O^{*} A 2.13$ & $467 \mathrm{C}>\mathrm{T} ; 742 \mathrm{C}>\mathrm{T}$ & P156L; R248C & 3 & $\mathrm{~A}_{2} \mathrm{~B}, \mathrm{~A}_{\mathrm{x}} \mathrm{B}$ & FJ998203 \\
\hline$A B O^{*} A 2 . n e w 5^{\mathrm{d}}(A 222)$ & $1054 \_1055$ insA & R352Qfs*39 & 1 & $\mathrm{~A}_{2}$ & KJ631752 \\
\hline$A B O^{*} A 3 . n e w 6^{\mathrm{d}}(A 312)$ & $280 \mathrm{~A}>\mathrm{T} ; 467 \mathrm{C}>\mathrm{T}$ & I94F; P156L & 1 & $\mathrm{~A}_{\mathrm{x}} \mathrm{B}$ & KM921804 \\
\hline$A B O^{*} A_{\mathrm{w}} \cdot n e w 7^{\mathrm{d}}(A x 22)$ & $389 \mathrm{~T}>\mathrm{C}$ & L130P & 1 & $A_{x}$ & KC866362 \\
\hline$A B O^{*} A E L .08$ & 467C $>\mathrm{T}$, 804dupG & P156L; F269Vfs*124 & $6(3)$ & Ael & HQ843789 \\
\hline$A B O^{*} B_{\mathrm{W}} \cdot n e w 9^{\mathrm{d}}(B 118)$ & $840 \mathrm{C}>\mathrm{T}$ & L280L & 1 & $\mathrm{~A}_{2} \mathrm{~B}$ & HQ843788 \\
\hline$A B O^{*} B_{\mathrm{W}} \cdot n e w 11^{\mathrm{d}}(B 120)$ & 484delG & V162OPA & 1 & $\mathrm{~B}_{\mathrm{el}}$ & KC526949 \\
\hline$A B O^{*} B 3.07$ & $410 \mathrm{C}>\mathrm{T}$ & A137V & 1 & $\mathrm{~B}_{3}$ & - \\
\hline$A B O^{*} B_{\mathrm{W}} \cdot$ new $12^{\mathrm{d}}\left(B_{\mathrm{w}} 27\right)$ & $910 A>G$ & S304G & 1 & $\mathrm{~B}_{\mathrm{x}}$ & JQ692625 \\
\hline$A B O^{*} B_{\mathrm{W}} \cdot$ new $14^{\mathrm{d}}\left(B_{\mathrm{w}} 35\right)$ & $737 A>G$ & Y246C & 1 & $\mathrm{~B}_{\mathrm{w}}$ & KM362861 \\
\hline$A B O^{*}$ B3.new $15^{\mathrm{d}}(B 314)$ & 3_4delG & M1Mfs* 18 & 1 & $\mathrm{AB}_{\mathrm{x}}$ & KC960560 \\
\hline$A B O^{*} B 3 . n e w 16^{\mathrm{d}}(B 315)$ & $928 \mathrm{C}>\mathrm{G}$ & L310V & 1 & $\mathrm{~B}_{\mathrm{w}}$ & KR021361 \\
\hline$A B O^{*} B_{\mathrm{W}} \cdot n e w 17^{\mathrm{d}}\left(B_{\mathrm{w}} 38\right)$ & $518 \mathrm{~T}>\mathrm{C}$ & L173P & 1 & $\mathrm{AB}_{\mathrm{w}}$ & KP245739 \\
\hline$A B O^{*} B_{\mathrm{W}} \cdot n e w 18^{\mathrm{d}}\left(B_{\mathrm{m}} 03\right)$ & $98 \mathrm{G}>\mathrm{C}$ & G33A & 2 & $\mathrm{~B}_{\mathrm{m}}$ & KC960559 \\
\hline$A B O^{*}$ cis $A B .05$ & $297 \mathrm{~A}>\mathrm{G} ; 526 \mathrm{C}>\mathrm{G} ; 657 \mathrm{C}>\mathrm{T}$; & R176G; G235S; L266M & 1 & $\mathrm{AB}_{\mathrm{w}}$ & - \\
\hline & $703 \mathrm{G}>\mathrm{A} ; 796 \mathrm{C}>\mathrm{A} ; 930 \mathrm{G}>\mathrm{A}$ & & & & \\
\hline
\end{tabular}

a The part of novel alleles of $A$ and $B$ firstly identified in our laboratory were named by ISBT, which were cited from Erythrogene (https://doi.org/10.1182/bloodadvances.2016001867). ${ }^{\mathrm{b}}$ The main nucleotide and amino acid changes of the $A B O^{*} A, A B O^{*} c i s A B$, and $A B O^{*} O$ alleles were compared to the consensus $A B O^{*} A 1.01 ; A B O^{*} \mathrm{~B}$ alleles were compared to $A B O^{*} B .01$ allele. ${ }^{\mathrm{c}}$ The number of the sample identified in this study. $R$, number of related family member. ${ }^{\mathrm{d}}$ The novel alleles firstly found in our laboratory are not named by ISBT currently, but have been named according to the nomenclature used in the dbRBC before [4]. ${ }^{\text {e }}$ The sequence was not submitted to the NCBI before.

rect sequencing of the entire $A B O$ coding region and flanking splicing sites, including 16 novel $A B O$ alleles. All of these alleles were classified as 5 groups: $16 A B O^{*} A$ alleles, $23 A B O^{*} B$ alleles, $4 A B O^{*} B A$ alleles, $4 A B O^{*} c i s A B$ alleles, and $1 A B O^{*} O$ alleles. $A B O^{*} A 2.08, A B O^{*} B A .02$, $A B O^{*} B A .04, A B O^{*} B_{W} .03$ alleles had a relatively high frequency (see www.karger.com/doi/10.1159/000501862; online suppl. Table S1). 


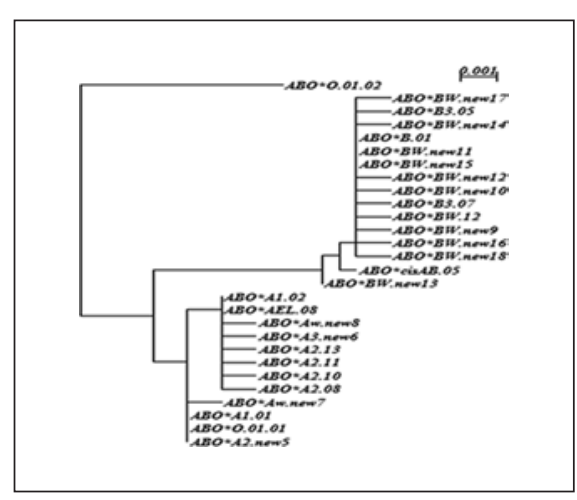

Fig. 1. The phylogenetic tree for 28 alleles ( 5 common alleles and 23 alleles identified in our laboratory) based on $\mathrm{ABO}$ gene sequences of exon 1-7.

\section{Sequence Characteristics of the Novel Alleles}

After haplotype sequence analysis of $A B O$ gene, 16 novel alleles were identified in variant samples (Table 2), and part of $A B O$ alleles have been named according to the nomenclature by ISBT. Most of the novel alleles had a nucleotide mutation in exon 6-7, resulting in an amino acid change in the catalytic region. Compared with the reference allele, two naturally occurring $A B O^{*} B$ alleles $\left(A B O^{*} B_{W}\right.$.new 11 and $A B O^{*} B_{3 .}$ new 15$)$ had a nucleotide deletion and $2 A B O^{*} A$ alleles $\left(A B O^{*} A E L .08\right.$ and $A B O^{*} A 2$. new5) had a single base insertion. All of them led to a frameshift to create a premature terminal codon at different position of GT enzyme. One interesting new mutation, $A B O^{*}$ cis $A B .05$, had a nucleotide reversion c. $803 \mathrm{C}>\mathrm{G}$ compared with $A B O^{*} B .01$ allele.

\section{Phylogenetic Tree}

A phylogenetic tree was constructed from a neighborjoining analysis of $28 A B O$ alleles (the common $A B O$ alleles and the subgroup alleles detected in our laboratory) based on the sequences of exon 1-7 (Fig. 1). Most branch lengths were very short and bootstrap values were small. It was clear that 28 alleles were classified into 3 distinct allelic lineages. Group I consisted of $A B O^{*} B$-related alleles and $A B O^{*}$ cis $A B$, while Group II consisted of $A B O^{*} A$ related alleles and $A B O^{*} 0.01 .01$. The $A B O^{*} 0.01 .02$ is a separate group.

The Analysis of the 3D Structures of the Mutation GTs from the Typical Novel Allele Mutations

Most of the novel alleles were point mutations located in the catalytic domain (Fig. 2A, B). Thus, 2 point mutations $\left(A B O^{*} A\right.$ : c. $389 \mathrm{~T}>\mathrm{C}$, p.L130P; $A B O^{*} B:$ c. $410 \mathrm{C}>\mathrm{T}$, p.A137V) and 1 deletion mutant allele $\left(A B O^{*} B:\right.$ c.484delG, p.V162OPA) were selected for analysis of the enzyme structure prediction. The overall structures of the point mutant GTs were predicted to be similar to that of the wild type. However, although the overall shapes of these enzymes were similar, the mutations were predicted to form the different hydrogen bonds with the other surrounding residues (Fig. 2C-F). In the wild-type GTA, amino acid residue L130 was predicted to form 2 hydrogen bonds with 2 surrounding residues (V127 and L134), as indicated in Figure 2C, while in the p.L130P mutant GTA, P130 also formed hydrogen bonds with these 2 amino acids, but the atoms and positions for forming the hydrogen bonds had changed significantly (Fig. 2D). The hydrogen bond distance was elongated and structural stability changed. Meanwhile, compared with the wild-type GTB, the p.A137V mutant GTB reduced one hydrogen bond with the surrounding amino acid residues. Four hydrogen bonds were reduced to 3 bonds, because the V137 mutation did not form a hydrogen bond with F141. The atoms and distances of the hydrogen bonds were different as well (Fig. 2E, F). For the deletion mutation allele, c.484delG formed C-terminally truncated enzymes, which mainly lead to the GTB structural changes and may not form effective active center (Fig. 2G).

\section{ABO Subgroup Phenotypes with the Other Molecular \\ Basis}

Another 75 variants were considered to have an unclear molecular basis because only normal sequences were detected in the analysis regions. Among these individuals, 32 samples were identifiable for infection, hematologic disorders, and other diseases. Thus, we presumed that the antigen or antibody changes in these individuals might be concerned with the diseases. Seven $A B O$ variants were concerned with the erythroid cell-specific regulatory element in the first intron of $A B O$ gene. The molecular genetic analyses were shown in our recently report [10]. The other 36 samples (including 4 individuals with pregnancy) without any diseases or other molecular basis might have an unclear molecular basis.

\section{Discussion}

In this study, $211 \mathrm{ABO}$ variants associated with discrepant $\mathrm{ABO}$ phenotype were investigated. However, there were only 136 individuals driving from the rare alleles with mutations distributed over the entire coding region of the ABO gene by PCR-SBT method. Thus, 48 distinctly rare $A B O$ alleles, including 16 novel subgroup alleles, were characterized. The detection of alleles was not only an important contribution for each individual case, but also a development of the blood group genetic factors. The availability of these rare $A B O$ alleles will present researchers with more information regarding the structure of $A B O$ genes. The sequences can be used to design new primers and oligonucleotide probes to aid 
Fig. 2. Ribbon diagrams of the wild-type GTs (GTA: PDB code, 1LZI and GTB: PDB code, 1LZ7) and the mutant GTs. A Structures of the wild-type GTA and the amino acid residue position with the novel point mutation. B Structures of the wild-type GTB and the amino acid residue position with the novel point mutation. C In the wild-type GTA, the side chain of L130 can form 2 hydrogen bonds with 2 surrounding residues (V127 and L134) indicated by the red dotted lines. D In the p.L130P mutant GTAs, the side chain of P130 can form different hydrogen bonds with these residues. E In the wild-type GTB, the side chain of A137 can form 4 hydrogen bonds with 4 surrounding residues (F133, H140, F141, and R168) indicated by the red dotted lines. F In the p.A137V mutant GTB, the side chain of V137 only can form another 3 different hydrogen bonds with these residues except the F141 amino acid. G The structural model of the mutant GTB with c.484del $G$ allele. The figures were generated using Pymol software.

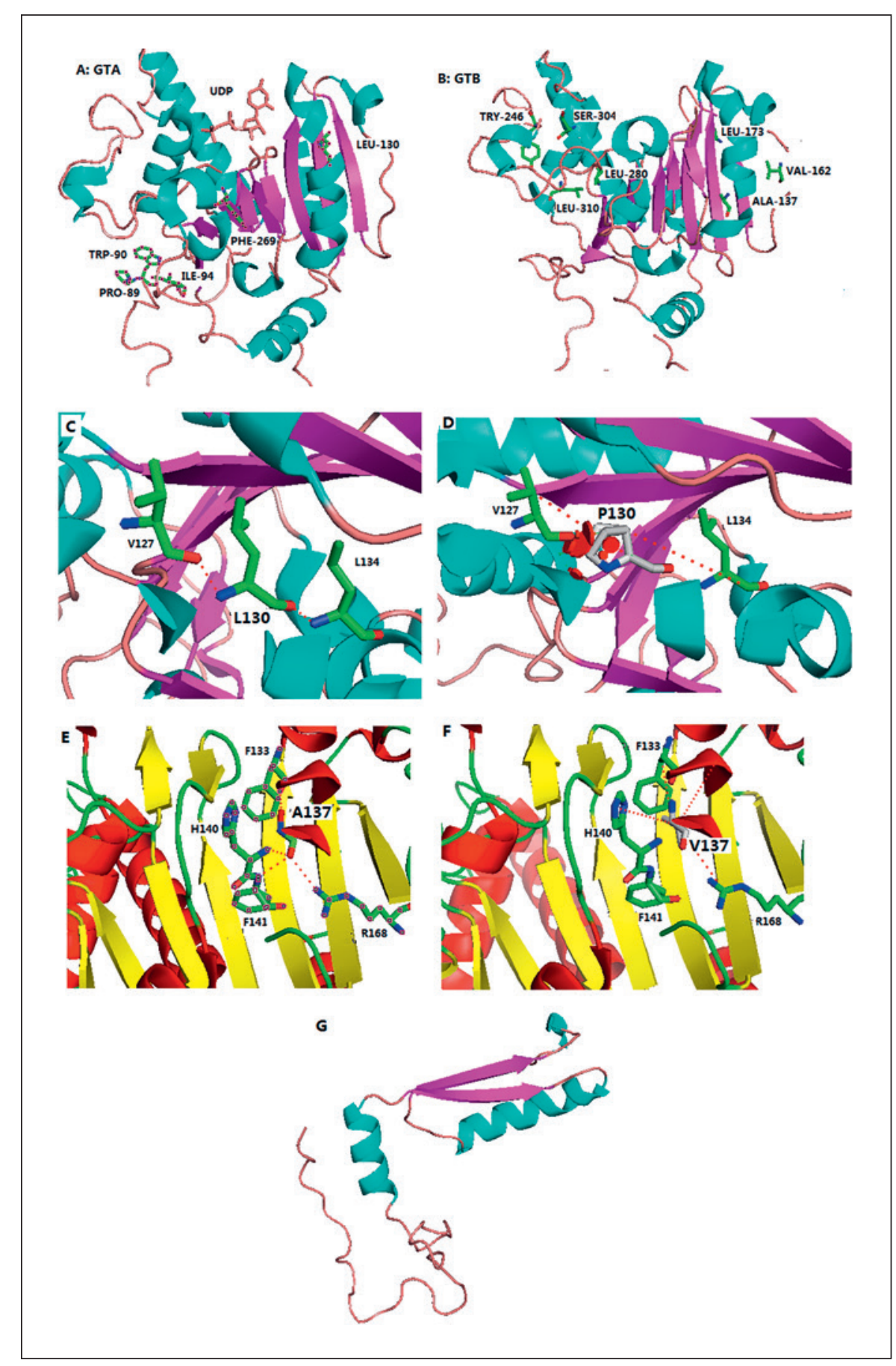

more exact $A B O$ typing, and the polymorphism data can be used for evolutionary studies as well.

Among these $\mathrm{ABO}$ variants, 80 samples were screened from about 0.7 million blood donors. However, the frequency of the rare alleles could not be accurately calculated because the samples tested were not collected by large-scale screening from random populations. Meanwhile, only the samples with obvious discrepant phenotype were collected for sequencing, so, many potential samples might be lost. For the low-frequency alleles (only
1 individual was found), the numbers detected also did not represent the true distribution. But according to our previous study, none of these mutations were found in 417 random samples, which suggested that the frequency of these mutations was $<0.12 \%$ in Chinese population [17].

From the distribution of alleles, $A B O^{*} A 2.08$ and $A B O * B A .02$ were the relatively predominant alleles. The number of rare $A B O^{*} B$ alleles (including $A B O^{*} B A ; 27$ $A B O^{*} B$ alleles) was a little higher than the $A B O^{*} A$ alleles 
(including $A B O^{*}$ cis $A B ; 20 A B O^{*} A$ alleles) in our study. Combining our previous studies on the distribution of $A B O$ blood group allele and the molecular basis of A2 subgroup before [15, 17], the type of the rare $A B O$ alleles in this study was little different from the results of the others [19]. It clearly indicated that the molecular basis of $\mathrm{ABO}$ variants had specific characteristics among the different populations. The best blood transfusion practices involving $\mathrm{ABO}$ variants will be based on the data and experience of specific populations. The amino acid changes had different properties and significant effects on the encoded protein. The nucleotide mutation occurred with high frequency (91.3\%) in exons 6 and 7 corresponding to the catalytic domain of GT, which is the crucial function part of the protein [20]. According to the $3 \mathrm{D}$ structure analysis, although the overall structure of the point mutant GTs was similar to the wild type, the mutation GTs expected that the amino acid residue replacement may change the numbers and the spatial distance of hydrogen bonds forming and induce the steric hindrance with the neighboring residues, resulting in decreasing the protein stability. Thus, the protein stability reduced by the hydrogen bond change may be a possible explanation of the changes of enzyme activity and properties, leading to the phenotypes of the mutants.

Except for the alleles with the point mutation, $2 \mathrm{nu}$ cleotide insertions c.804 dupG (ABO*AEL.08) and c.1054_1055 insA (A222) were identified with Ael and A2 phenotypes, respectively, resulting in a frame shift to p.F269Vfs*124 and p.R352Qfs*39 in GTAs. c.1054_1055insA was added, an additional 39 amino acids, to the enzyme before translation halted, whose features were similar to the c.1061delC $\left(A B O^{*} A 2.01\right)$ [5]. In addition, 2 nucleotide deletion c.3_4delG $\left(A B O^{*} B 3\right.$. new15) and c.484delG $\left(A B O^{*} B_{W}\right.$. new 11$)$ were detected with $\mathrm{ABx}$ and Bel phenotypes, respectively, resulting in a frameshift (p.M1Mfs*18 and p.V162OPA) for a premature termination in GTBs. Premature termination codons were generally considered to form production of a truncated and inactive protein [21]. The 3D structure of the mutant with c.484delG also predicted that these deletion mutations result in the inability to form a complete protein. But very weak antigens were detected in the individual by an adsorption-elution test. Previous studies showed that several $A B O$ subgroup alleles predicted to code for $\mathrm{C}$-terminally truncated enzymes had been described in individuals with weak A phenotypes as well $[22,23]$. Thus, the reductions of activity in these cases might be explained by partial lack of the catalytically active site. Remarkably, the individual with $A B O^{*} B_{3}$.new15 (c.3_4delG), leading to an N-terminally truncated B transferase (p.M1Mfs*18), showed agglutination strength $1+$ with anti-B. A possible explanation for this result was that another Met downstream of the premature termination codon might be initiated as the translation start codon, being expected to produce an $\mathrm{N}$-terminally shortened polypeptide, which may be capable of producing GTs with reduced enzyme activity [24].

All the genomic sequence data were useful for evaluating the genetic diversity and for lineage definition. Seltsam et al. reported that the intron-based phylogenetic analysis revealed 5 main lineages: $A B O^{*} A, A B O^{*} B$, $A B O^{*} O 01, A B O^{*} O .02$, and $A B O^{*} O 03$ [18]. In this study, we constructed phylogenetic tree for the common alleles and the subgroup alleles found in our laboratory according to the exon 1-7 nucleotide sequences. This finding may enrich the knowledge of coding region in the $A B O$ evolution. The figure clearly revealed that there were three distinct allelic lineages, $A B O^{*} A, A B O^{*} B$, and $A B O^{*}$ O.01.02. The $A B O^{*} O .01 .01$ alleles were included in the $A$-related group, which indicated that the relationship between $A$ and $O$ alleles was close. The Cis $A B$ belonged to $A B O^{*} B$ allelic lineage, indicating that the similarity between $C i s A B$ and $A B O^{*} B$ was higher than the $A B O^{*} A$. This relationship also indicated that $C i s A B$ was likely to be derived from the antisense mutation of $A B O^{*} B$ allele. However, understanding the evolutionary history of the $A B O$ locus is complicated because of the different levels of nucleotide diversity. The phylogenetic analysis may change by using different region sequences and the major allelic lineages were different.

In our study, another 75 variants were considered to have another molecular basis. In the sequence of the entire $A B O$ coding region and flanking splicing sites, we did not find any changes by the PCR-SBT method. The phenotype changes of 32 patient samples might be due to the diseases $[25,26]$. The erythroid cell-specific regulatory element in the first intron of $A B O$ gene also played an important role in the antigen differential expression. But the molecular mechanism underlying the other samples still remains largely unknown. We presumed that the other transcriptional regulatory elements and methylation might affect the transcriptional activity of the $A B O$ gene $[27,28]$. Further studies will be needed to assess the impact of the other regulatory elements on $\mathrm{ABO}$ transferase function.

In summary, we investigated the molecular basis of 211 Chinese $\mathrm{ABO}$ variants. 48 rare $A B O$ alleles, including 16 novel alleles, were characterized and their genetic diversity was analyzed. The effect of protein stability change induced by the mutation was a potential cause of the $A B O$ subgroup. In the practical work, our study may be beneficial for making more appropriate transfusion strategies for certain populations and thus help to improve the blood safety. 


\section{Acknowledgements}

This work was supported by the Natural Science Research Foundation of Zhejiang Province (LY17H080003), the National Natural Science Foundation of China (81902137), and the Medical Science Research Foundation of Zhejiang Province (2016RCB006, 2017KY315, WKJ-ZJ-1608). This work was sponsored by Zhejiang Provincial Program for the Cultivation of High-Level Innovative Health Talents.

\section{Statement of Ethics}

Informed consent was obtained, and the study was approved by the local Ethics Committee of the Blood Center of Zhejiang Province.

\section{Disclosure Statement}

The authors have no conflicts of interest to declare.

\section{References}

1 Storry JR, Olsson ML. The ABO blood group system revisited: a review and update. Immunohematology. 2009;25(2):48-59.

2 Storry JR. Nomenclature for red blood cell blood group alleles. Transfusion. 2013 Nov; 53(11 Suppl 2):2844-6.

3 Möller M, Jöud M, Storry JR, Olsson ML. Erythrogene: a database for in-depth analysis of the extensive variation in 36 blood group systems in the 1000 Genomes Project. Blood Adv. 2016 Dec;1(3):240-9.

4 Patnaik SK, Helmberg W, Blumenfeld OO. BGMUT: NCBI dbRBC database of allelic variations of genes encoding antigens of blood group systems. Nucleic Acids Res. 2012 Jan;40(Database issue):D1023-9.

5 Hult AK, Yazer MH, Jørgensen R, Hellberg A, Hustinx H, Peyrard T, et al. Weak A phenotypes associated with novel $\mathrm{ABO}$ alleles carrying the A2-related 1061C deletion and various missense substitutions. Transfusion. $2010 \mathrm{Jul}$; 50(7):1471-86.

6 Xiang D, Liu X, Guo ZH. Study on ABO subgroup in Chinese population in Shanghai area. Chin J Blood Transfus. 2006;19(1):25-6.

7 Yamamoto F, Clausen H, White T, Marken J, Hakomori S. Molecular genetic basis of the histo-blood group ABO system. Nature. 1990 May;345(6272):229-33.

8 Cai X, Li F, Lei H, Qu S, Qian C, Xiang D, et al. p.R180C mutation of glycosyltransferase $B$ leads to B subgroup, an in vitro and in silico study. Vox Sang. 2018 May;113(5):476-84.

9 Seltsam A, Blasczyk R. Missense mutations outside the catalytic domain of the ABO glycosyltransferase can cause weak blood group A and B phenotypes. Transfusion. 2005 Oct; 45(10):1663-9.

10 Ying Y, Hong X, Xu X, Ma K, He J, Zhu F. A novel mutation $+5904 \mathrm{C}[\{\mathrm{GT}\}] \mathrm{T}$ of RUNX1 site in the erythroid cell-specific regulatory element decreases the $\mathrm{ABO}$ antigen expression in Chinese population. Vox Sang. 2018 Jul; 113(6):594-600.
11 Sano R, Kuboya E, Nakajima T, Takahashi Y, Takahashi K, Kubo R, et al. A 3.0-kb deletion including an erythroid cell-specific regulatory element in intron 1 of the ABO blood group gene in an individual with the $\mathrm{Bm}$ phenotype. Vox Sang. 2015 Apr;108(3):310-3.

12 Sano R, Nakajima T, Takahashi K, Kubo R, Yazawa S, Kominato Y. The 3 ' flanking region of the human ABO histo-blood group gene is involved in negative regulation of gene expression. Leg Med (Tokyo). 2011 Jan;13(1): 22-9.

13 Takahashi Y, Isa K, Sano R, Nakajima T, Kubo $\mathrm{R}$, Takahashi $\mathrm{K}$, et al. Presence of nucleotide substitutions in transcriptional regulatory elements such as the erythroid cell-specific enhancer-like element and the $\mathrm{ABO}$ promoter in individuals with phenotypes A3 and B3, respectively. Vox Sang. 2014 Aug;107(2):17180.

14 Roback J. AABB technical manual. New York. Bethesda: American Association of Blood Banks; 2008. pp. 1-9

15 Ying Y, Hong X, Xu X, Liu Y, Lan X, Ma K, et al. Serological characteristic and molecular basis of A2 subgroup in the Chinese population. Transfus Apheresis Sci. 2013 Feb;48(1): 67-74.

16 Ying Y, Chen S, Ma K, Hong X, Zhu F. Identification of a novel B allele with missense mutation (c.98G $[\{G T\}] C$ ) in the ABO gene. Transfusion. 2017 Jan;57(1):219-20.

17 Zhu F, Tao S, Xu X, Ying Y, Hong X, Zhu H, et al. Distribution of ABO blood group allele and identification of three novel alleles in the Chinese Han population. Vox Sang. 2010 May;98(4):554-9.

18 Seltsam A, Hallensleben M, Kollmann A, Blasczyk R. The nature of diversity and diversification at the ABO locus. Blood. 2003 Oct; 102(8):3035-42.

19 Cai X, Jin S, Li X, et al. Molecular genetic analysis of ABO blood group variations reveals 28 novel ABO subgroup alleles. Transfusion. 2013;53(11 Suppl 2):2810-6.

20 Seltsam A, Das Gupta C, Bade-Doeding C, Blasczyk R. A weak blood group A phenotype caused by a translation-initiator mutation in the ABO gene. Transfusion. 2006 Mar;46(3): 434-40.
21 Wilkinson MF, Shyu AB. RNA surveillance by nuclear scanning? Nat Cell Biol. 2002 Jun; 4(6):E144-7.

22 Hosseini-Maaf B, Irshaid NM, Hellberg A, Wagner T, Levene C, Hustinx $\mathrm{H}$, et al. New and unusual $\mathrm{O}$ alleles at the $\mathrm{ABO}$ locus are implicated in unexpected blood group phenotypes. Transfusion. 2005 Jan;45(1):70-81.

23 Ogasawara K, Yabe R, Uchikawa M, Nakata K, Watanabe J, Takahashi Y, et al. Recombination and gene conversion-like events may contribute to $\mathrm{ABO}$ gene diversity causing various phenotypes. Immunogenetics. $2001 \mathrm{Apr}$; 53(3):190-9.

24 Hata Y, Kominato Y, Takizawa H, Tabata S, Michino J, Nishino $\mathrm{K}$, et al. Transcription starting from an alternative promoter leads to the expression of the human $\mathrm{ABO}$ histoblood group antigen. Transfusion. 2003 May; 43(5):656-62.

25 Garratty G. Blood group antigens as tumor markers, parasitic/bacterial/viral receptors, and their association with immunologically important proteins. Immunol Invest. 1995 Jan-Feb;24(1-2):213-32.

26 Chihara Y, Sugano K, Kobayashi A, Kanai Y, Yamamoto H, Nakazono M, et al. Loss of blood group A antigen expression in bladder cancer caused by allelic loss and/or methylation of the ABO gene. Lab Invest. $2005 \mathrm{Jul}$; 85(7):895-907.

27 Kominato Y, Hata Y, Takizawa H, Matsumoto K, Yasui K, Tsukada J, et al. Alternative promoter identified between a hypermethylated upstream region of repetitive elements and a CpG island in human ABO histo-blood group genes. J Biol Chem. 2002 Oct;277(40): 37936-48.

28 Sano R, Nakajima T, Takahashi Y, Kubo R, Kobayashi M, Takahashi K, et al. Epithelial expression of human $\mathrm{ABO}$ blood group genes is dependent upon a downstream regulatory element functioning through an epithelial cell-specific transcription factor, Elf5. J Biol Chem. 2016 Oct;291(43):22594-606. 